\title{
Performance Analysis of Vehicular Optical Camera Communications: Roadmap to uRLLC
}

\author{
Amirul Islam, Leila Musavian, and Nikolaos Thomos \\ CSEE, University of Essex, UK. \\ Email: \{amirul.islam, leila.musavian, nthomos\}@essex.ac.uk
}

\begin{abstract}
In this paper, we analyze the performance of vehicular optical camera communication (OCC) towards ultrareliable and low latency communications (uRLLC). The employed vehicular OCC model uses light-emitting diodes (LED) as transmitter and camera as receiver. In particular, we investigate the performance of the proposed system in terms of bit error rate (BER), spectral efficiency, and transmission latency at different inter-vehicular distances and angle of incidences (AoI). Further, we investigate the use of adaptive modulation to improve the spectral efficiency. From our analysis, we note that by satisfying a given target BER, higher spectral efficiency and lower latency can be achieved through adjusting the AoI towards the smaller degrees and switching into the suitable modulation order. Finally, we verify the results through simulations, which show that OCC can ensure ultra-low latency as well as satisfy the reliability requirements in automotive vehicles.
\end{abstract}

\section{INTRODUCTION}

Automotive vehicles (AVs) are emerging as the revolution in future smart cities and are considered as one of the main transformative technologies in intelligent transportation systems (ITS). We are witnessing an unparallel increase of the number of vehicles and vehicle-assisting infrastructures resulting in more traffic congestions, road causalities, and overall less traffic safety. Communication between AVs can help improving the traffic safety and enhancing the overall driving experience by facilitating new service features, such as collision avoidance and autonomous driving [1]. Although several AVs services, such as lane changing alert [2] or automotive braking system [3], have already been deployed, mission-critical services, e.g., collision avoidance, automotive driving, are still posing significant challenges in vehicular networks. The efficiency of ITSs depends on the availability of reliable communication links within the shortest possible time that is characterized by uRLLC. Therefore, future AVs will require uRLLC to exchange their internal or surrounding information, e.g., speed, next action, and position, with each other effectively and operate the AVs reliably. However, achieving uRLLC is one of the major challenges in future vehicular networks [4], [5].

For enabling uRLLC in ITSs, existing methods, such as [6], [7] reflect on delay minimization, vehicle clustering, and excess queue length evaluation. Specifically, in [6], the vehicular network transmission power is minimized by grouping vehicles into clusters modelling reliability as queuing delay violation probability. A joint resource allocation and power control algorithm is proposed to maximize the vehicle-tovehicle (V2V) sum rate with latency and reliability constraints in [7]. Edge computing is also considered as an attractive solution to minimize latency that processes the requested tasks locally, without relying on remote servers [8], [9]. The above systems enable uRLLC, either using radio frequency (RF) communication or cellular systems with central base stations (BS), servers or edge servers. However, BSs can become overloaded with the frequent requested AV tasks because they have limited computational resources and follow centralized resource management. Moreover, RF channels are prone to channel fading, noise, and interference, which render them inappropriate for uRLLC.

On the other hand, recently, visible light communications (VLC) have attracted tremendous attention as a potential alternative to RF communication [10], [11]. Different from RF systems, VLC uses LEDs as transmitters and photodiodes (PD) or image sensors as receivers. VLC systems using PD as the receiver are called light fidelity (LiFi) and those employ image sensors are called OCC. VLC offers several significant advantages over RF-based systems including licensefree access spectrum, longer lifespans, less implementation cost, and enhanced security having the line of sight (LOS) properties [12]. More importantly, VLC systems do not pose any potential harm to human bodies or eyes and they do not create electromagnetic interference (EMI).

In traditional VLC, the receiver often consists of a nonimaging device, i.e., PD, and its performance is limited by the trade-off between transmission range and signal reception. Different from PD-based systems, OCC can spatially separate and process different sources independently on its image plane, which enables the receiver to discard noise sources, e.g., Sun, streetlights, other light sources, and focuses mainly on the pixels to which the LEDs strikes [13]. This ability ensures interference-free, reliable, and secure communication even at the outdoor environment. Table I summarizes the main characteristics of OCC, PD and RF communication systems, which shows that OCC suffers from almost negligible interference and consumes less power than RF. Besides, OCC supports almost 20 times longer distance than the PD-based systems. Although having low data rate, OCC can be a better alternative to the congested and saturated RF system due to its negligible noise and interference characteristics. The revolutionary advancements in OCC have made the technology as a promising mechanism for AVs communication [13], [14]. However, OCC can face challenges due to its LOS requirements for communication, i.e., communication links can be obstructed by objects or bad weather conditions. 
TABLE I

COMPARISON BETWEEN OCC, PD, AND RF

\begin{tabular}{|c|c|c|c|}
\hline \multirow{2}{*}{ Parameter } & \multicolumn{2}{|c|}{ OWC } & \multirow{2}{*}{$\mathbf{R F}$} \\
\hline & $\mathbf{O C C}$ & PD & \\
\hline Bandwidth of the carrier & Unlimited $(400-700) \mathrm{nm}$ & Unlimited $(400-700) \mathrm{nm}$ & $300 \mathrm{GHz}$ (saturated and regulated) \\
\hline EMI and hazard & No & No & Yes \\
\hline Transmitter & LED & LED or Laser diode (LD) & Antenna \\
\hline Receiver & Camera & PD & Antenna \\
\hline Power consumption & Relatively low & Relatively higher than OCC & Medium \\
\hline Interference level & Negligible & Low & Very high \\
\hline Communication distance & $200 \mathrm{~m}$ & $10 \mathrm{~m}$ & More than $100 \mathrm{~km}$ using Microwave \\
\hline Environmental effect & No & Indoor: No, Outdoor: Yes & Yes \\
\hline Noise & No & Sun and ambient light sources & All electrical and electronic appliances \\
\hline Security & High & High & Low \\
\hline Data rate & $54 \mathrm{Mbps}$ & $\begin{array}{l}10 \text { Gbps using LED and } 100 \text { Gbps } \\
\text { using LD }\end{array}$ & 6 Gbps (IEEE 802.11ad at 60GHz) \\
\hline Main purpose & $\begin{array}{l}\text { Illumination, communication, and } \\
\text { localization }\end{array}$ & Illumination and communication & Communication and positioning \\
\hline Limitation & Low data rate & $\begin{array}{l}\text { Short distance, no mobility guaranty, } \\
\text { not suitable for outdoor }\end{array}$ & Interference \\
\hline
\end{tabular}

To the best of our knowledge, this is the first OCC-based vehicular system that focuses on uRLLC aspects. In this paper, we introduce a novel low latency V2V communications framework that ensures ultra-reliability using OCC. The proposed system is fully decentralized and each vehicle processes the communicated information individually. In terms of latency, we only consider transmission latency, as a small amount of data is processed in our system that is related to the action or safety information, and hence, the computational latency is negligible. To improve the efficiency of OCC-based communication, we use an adaptive modulation scheme. By increasing the modulation order, higher spectral efficiency and lower latency can be achieved. In our evaluation, we consider satisfying the target BER as an indication of reliability in our system. If the reliability requirement for a certain modulation scheme is not met, the system can reduce the AoI at the receiver to ensure uRLLC. Finally, we analyze the performance of the proposed system in terms of BER, spectral efficiency, transmission latency for various inter-vehicular distances and AoIs of LED lights at the receiver. The major contributions of this paper can be summarized as follows:

- This is the first study that formulates the communication link performance with adaptive modulation scheme to examine whether OCC is suitable for employing uRLLC in automotive vehicles.

- We provide a mathematical framework to model the OCC channel in order to find out the probability of errors, achievable spectral efficiency, and transmission latency as a function of inter-vehicular distances and AoIs while considering the adaptive modulation.

- We investigate how to achieve uRLLC by introducing a mechanism of varying the AoI at the receiver vehicle when the transmitter changes the modulation scheme depending on the size of the transmitting data.

\section{SYSTEM MODEL}

\section{A. System Overview}

Let us consider two vehicles that communicate with each other as shown in Fig. 1; one being the transmitter vehicle
(TV) denoted by $T_{i}$ and the other being the receiver vehicle (RV) denoted by $R_{j}$. In our system, the LED lights located at the back side of TV is the transmitter, and the high-speed camera (also known as image sensor and has a frame rate of $1000 \mathrm{fps})$ located at the front side of RV, is the receiver. We denote the distance between $T_{i}$ and $R_{j}$ by $d_{i j}$. The communicated information between the vehicles is vehicle's internal information, e.g., speed, next action, position, and/or other safety and action-related information from the transmitter. In our system, the camera performs two simultaneous functions. Firstly, it measures $d_{i j}$. Secondly, the camera in the RV decodes the signal information received from the LED transmitters. Intensity modulation with direct detection (IM/DD) is adopted by the transmitter in which the desired waveform is modulated onto the instantaneous optical power of the LED lights.

\section{B. Optical Channel Model}

In our analysis, we assume an un-interrupted LOS link between the transmitter LED lights and the camera of the receiver. This ensures obstruction free and continuous communication. The light signal transmitted from the LED arrays is received by an image sensor in the RV which lies within its field of view (FoV). Then, the radiated signal passes through an optical filter and a lens to ensure that maximum light falls within the FoV of the receiver. Depending on the link conditions, the VLC channel is either a flat fading channel or a diffuse channel. Generally, VLC channel has two types of light propagation, namely, LOS component resulting from direct light propagation to the receiver and diffuse components resulting from the light reflections from other reflection surfaces or vehicles. Usually, the energy of diffuse components are much lower than the energy of the LOS component and, therefore, the latter is neglected in this paper. As a result, the optical wireless LOS channel DC gain is modelled as [15]:

$$
H_{i j}(\theta, t)=\left\{\begin{array}{cc}
\frac{A_{\text {eff }}(\theta)}{d_{i j}^{2}(t)} R(\phi), & 0 \leq \theta \leq \theta_{l} \\
0, & \theta>\theta_{l}
\end{array}\right.
$$




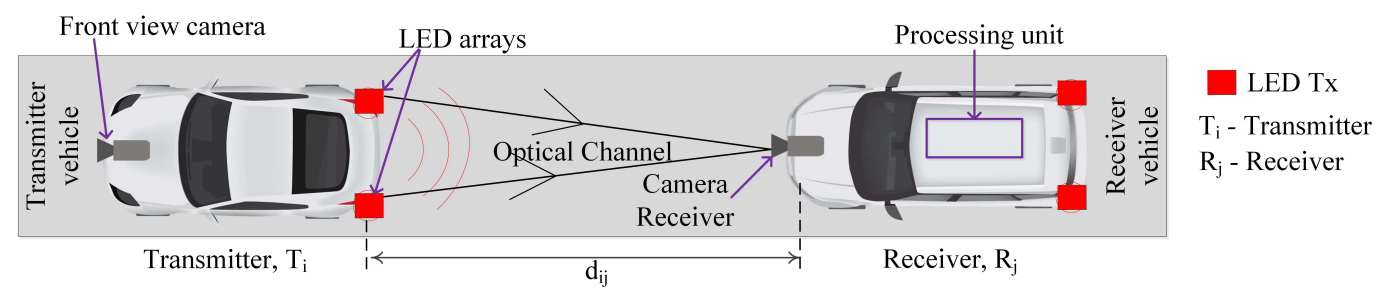

Fig. 1. Proposed system model of vehicular optical camera communication.

where $A_{\text {eff }}(\theta)$ is the effective signal collection area of the image sensor, $\theta$ is the AoI, $\phi$ is the angle of irradiance with respect to the emitter, $R(\phi)$ is the transmitter radiant intensity, $\theta_{l}$ denotes the FoV of the image sensor lens, and finally, $t$ is time frame index. The distance $d_{i j}(t)$ can be expressed as [14]

$$
d_{i j}(t)=\frac{f}{a} \cdot \frac{D}{p(t)},
$$

where $D$ is the distance between the left and right LED array units, $f$ is the lens focal length, $p(t)$ is the distance in terms of number of pixels between the left and right LED array units on the captured image, and $a$ is the image pixel size. The inter-relation between the distance calculation parameters is illustrated in Fig. 2(a).

Regarding the above parameters: $D$ is sent from the TV to $\mathrm{RV}$, and $f$ and $a$ are known values, such as $15 \mathrm{~mm}$ and $7.5 \mu \mathrm{m}$, in this system. The value of $p(t)$ can be obtained via simple image processing techniques or calculating the pixel values using data pointer.

However, the DC gain can accurately be computed by considering the LOS propagation. For this, we follow the link geometry as shown in Fig. 2(b). As LED light usually has the Lambertian radiation pattern, the light emission from the LED transmitters can be modeled using a generalized Lambertian radiant intensity [14], [16]

$$
R(\phi)=\frac{(m+1)}{2 \pi} \cos ^{m}(\phi),
$$

where $m$ is the order of Lambertian emission which is related to the LED semiangle at half luminance $\left(\Phi_{1 / 2}\right)$, given by [15]:

$$
m=\frac{-\ln (2)}{\ln \left(\cos \left(\Phi_{1 / 2}\right)\right)} .
$$

$A_{\text {eff }}(\theta)$ in (1) of the projected image on the image sensor can be expressed as [15]

$$
A_{\text {eff }}(\theta)=\left\{\begin{array}{cc}
A T_{s}(\theta) g \cos (\theta), & 0 \leq \theta \leq \theta_{l} \\
0, & \theta>\theta_{l}
\end{array}\right.
$$

where $A$ is the area of the entrance pupil of the camera lens, $T_{s}(\theta)$ is the signal transmittance of the optical filter, and $g$ is the gain of the lens, which is given by

$$
g=\frac{n^{2}}{\sin ^{2}\left(\theta_{l}\right)},
$$

where $n$ corresponds to the internal refractive index of an ideal lens. Taking into account (3) and (5), (1) can be written as follows:

$H_{i j}(\theta, t)=\left\{\begin{array}{cc}\frac{(m+1) A}{2 \pi d_{i j}^{2}(t)} \cos ^{m}(\phi) g T_{s}(\theta) \cos (\theta), & 0 \leq \theta \leq \theta_{l} \\ 0 . & \theta>\theta_{l}\end{array}\right.$
From (7), we observe that if $A$ and $g$ are fixed for an image sensor, the channel power gain $H_{i j}(\theta, t)$ can be increased by either (a) decreasing the distance, $d_{i j}(t)$ and/or (b) increasing the collection area, i.e., by decreasing the AoI of the camera lens. Lower AoI of the camera lens means the strength of light beam will be stronger on the image sensor, which in turns, will increase the channel power gain. Alternatively, higher AoI reduces the $H_{i j}(\theta, t)$ as the LED light beam will spread out at the wide angle of the camera lens. So, maintaining narrower AoI at the receiver will provide improved performance because of having higher gain.

Finally, the received optical power $P_{\mathrm{r}}(\theta, t)$ can be derived from the optical transmitted power $P_{\mathrm{t}}$ from the LEDs as

$$
P_{\mathrm{r}}(\theta, t)=P_{\mathrm{t}} H_{i j}(\theta, t) .
$$

\section{Analysis of Adaptive Modulation And AoI ADJUSTMENT}

Motivated by the trade-off between the order of modulation and the achieved BER, we consider adaptive modulation that permits us to adopt modulation order by satisfying minimum BER for the system. Moreover, adaptive modulation offers improved spectral efficiency. It is worth to note that the adjustment of modulation depends on the road scenarios. At normal conditions, when there is nothing to communicate, RV maintains the wider AoI to understand the whole scenario of the road. If the TV wants to transmit any critical information, it chooses a higher modulation based on the size of the transmitted data. On the receiver side, if the RV notices any sudden change in the TV transmitted signal and fails to decode it using the current modulation scheme, the RV switches to another modulation from the chosen limited modulation set. In the meantime, RV decreases the AoI of the camera lens to focus on the LED transmitter and decodes the transmitted signal within the shortest possible time.

In order to analyze the system performance in terms of BER, spectral efficiency, and latency, we first need to formulate the signal-to-noise ratio (SNR) of the optical link. We consider SNR as a measure of communication link quality of the signal transmission. Therefore, according to [16], the received SNR $\gamma(\theta, t)$ of visible light link can be expressed by

$$
\gamma(\theta, t)=\frac{P_{\mathrm{r}}^{2}(\theta, t)}{\sigma_{\text {total }}^{2}(\theta, t)}=\frac{P_{\mathrm{t}}^{2} H_{i j}^{2}(\theta, t)}{\sigma_{\text {total }}^{2}(\theta, t)},
$$

where $\sigma_{\text {total }}^{2}(\theta, t)$ denotes total noise power and can be expressed as

$$
\sigma_{\text {total }}^{2}(\theta, t)=\sigma_{\text {shot }}^{2}(\theta, t)+\sigma_{\text {thermal }}^{2} .
$$




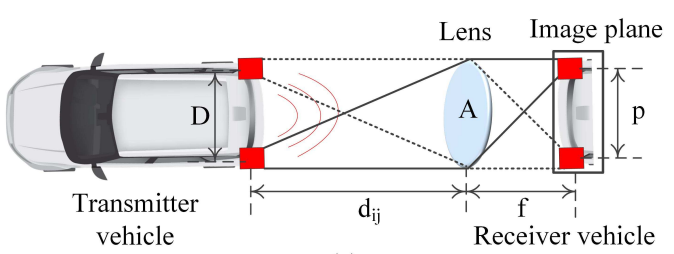

(a)

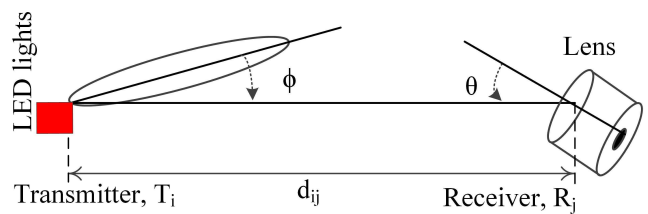

(b)

Fig. 2. (a) Inter-vehicular distance measurement [14] and (b) LOS channel model of OCC.

While shot-noise variance $\sigma_{\text {shot }}^{2}(\theta, t)$ is given by

$$
\sigma_{\text {shot }}^{2}(\theta, t)=2 q B\left(s P_{\mathrm{r}}(\theta, t)+I_{\mathrm{bg}} I_{2} P_{n}\right),
$$

where $q$ is the electronic charge, $B$ is the equivalent noise bandwidth, $I_{\mathrm{bg}}$ is the background current, $P_{n}$ is the noise power $\left(I_{\mathrm{amp}} / R_{b}\right), I_{\mathrm{amp}}$ is the amplifier current, $R_{b}$ is the data rate, and $I_{2}$ is the noise bandwidth factor for a rectangular transmitter pulse shape.

The thermal noise variance in (9) is given by

$$
\sigma_{\text {thermal }}^{2}=\frac{8 \pi k T}{G} I_{2} B^{2} C_{f} A+\frac{16 \pi^{2} k T \Gamma}{g_{m}} I_{3} B^{3} C_{f}^{2} A^{2},
$$

where $k$ is Boltzmanns constant, $T$ is absolute temperature, $G$ is the open-loop voltage gain, $C_{f}$ is the fixed capacitance of the image sensor per unit area, $g_{m}$ is the FET transconductance, $\Gamma$ is the FET channel noise factor, and $I_{3}$ is the noise bandwidth factor.

From (7) and (9), we can see that the received SNR depends on both AoI and the distance between the transmitter and receiver. Therefore, we can control the SNR by modifying the AoI and $d_{i j}(\theta, t)$.

In the proposed system, we use adaptive modulation scheme with the combination of binary phase shift keying (BPSK), Mary quadrature amplitude modulation (M-QAM), and M-ary phase shift keying (M-PSK) as example, but other modulation schemes can be used as well. For the performance analysis, we estimate the achievable BER by each modulation scheme using the formulas found in [17].

We should note that channel capacity (measured in bits/sec) of a camera based communication system depends on the employed modulation scheme as has been shown in [12] where capacity is expressed as

$$
C(\theta, t)=W_{\mathrm{fps}} \cdot W_{\mathrm{s}}(t) \cdot \log _{2}(M(\theta, t)),
$$

where $W_{\mathrm{fps}}$ is the camera-frame rate in fps, $W_{\mathrm{s}}(t)$ is the spatial-bandwidth (14), which can also be denoted by the number of information carrying pixels per camera image frame, and $M(\theta, t)$ is constellation size. $\log _{2}(M(\theta, t))$ is the spectral efficiency which depends on the modulation scheme, e,g, 1 for BPSK, 2 for 4-QAM. The spatial bandwidth $W_{\mathrm{s}}(t)$ can be defined by

$$
W_{s}(t)=N_{\text {LEDs }} \cdot N_{\text {row }}(t),
$$

where $N_{\text {LEDs }}$ is the number of LEDs at each row of the transmitter and $N_{\text {row }}$ represents the captured number of row pixel lines in each frame. Considering the operation of the

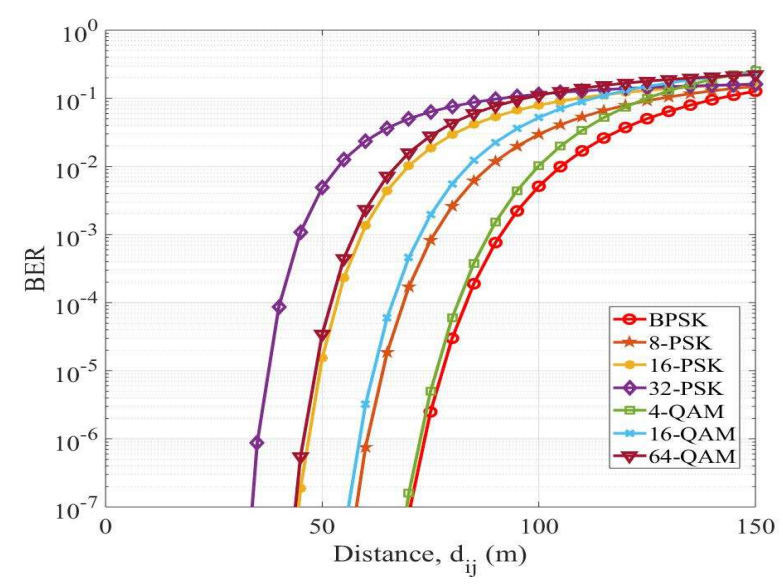

Fig. 3. BER vs distance for different modulation schemes.

rolling shutter camera, the actual number of samples (pixel rows) can be expressed as follows:

$$
N_{\text {row }}(t)=w \cdot \frac{L_{\text {size }}}{2 \tan \left(\frac{\theta_{l}}{2}\right) \cdot d_{i j}(t)},
$$

where $w$ is the image width (in case the rolling axis is along the width of the image sensor), and $L_{\text {size }}$ is the size of LED lights in $\mathrm{cm}^{2}$.

Hence, the overall end-to-end latency can be found as

$$
\tau(\theta, t)=\frac{L}{C(\theta, t)},
$$

where $\tau(\theta, t)$ represents transmission latency which includes the downlink latency only and $L$ is the packet size in bits. Please recall that, we neglect the computational latency as in our system small amount of data should be processed.

Since, the goal of the system is to avoid critical conditions, i.e., avoid collision between vehicles, a minimum distance has to be maintained between the vehicles. However, with the increase of the distance between the vehicles deteriorates the quality of the communication. Specifically, increasing the distance beyond a threshold would lead uRLLC conditions to be violated. So, in order to maintain uRLLC, we can vary the modulation order at the transmitter depending on the size of the transmitting data and the AoIs at the RV to satisfy the target BER.

\section{Simulation Results And Discussion}

In this section, we evaluate the proposed system for different performance metrics to get a better understanding of 
TABLE II

SIMULATION PARAMETERS

\begin{tabular}{|l|l|l|l|}
\hline \multicolumn{1}{|c|}{ Parameter, Notation } & \multicolumn{1}{c|}{ Value } & \multicolumn{1}{c|}{ Parameter, Notation } & \multicolumn{1}{c|}{ Value } \\
\hline Angle of irradiance w.r.t. the emitter, $\phi$ & $70^{\circ}$ & Boltzmanns constant, $k$ & $1.3807 \times 10^{-23}$ \\
\hline Semi-angle at half luminance of the LED, $\Phi_{1 / 2}$ & $60^{\circ}$ & Absolute temperature, $T$ & $298 \mathrm{~K}$ \\
\hline Inter-vehicular distance, $d_{i j}$ & $(0-150) \mathrm{m}$ & Open loop voltage gain, $G$ & 10 \\
\hline AoI w.r.t. the receiver axis, $\theta$ & $0^{\circ}$ to $90^{\circ}$ & Fixed capacitance, $C_{f}$ & 1.5 \\
\hline FOV of the camera lens, $\theta_{l}$ & $90^{\circ}$ & FET channel noise factor, $\Gamma$ & $30 \mathrm{~ms}$ \\
\hline Image sensor physical area, $A$ & $10 \mathrm{~cm}^{2}$ & FET trans-conductance, $g_{m}$ & 0.0868 \\
\hline Transmission efficiency of the optical filter, $T_{s}$ & 1 & Noise bandwidth factor, $I_{3}$ & $8,16,32$ for M-PSK and \\
\hline Refractive index of concentrator/lens, $n$ & 1.5 & Constellation size, $M$ & $4,16,64$ for M-QAM \\
\hline Concentrator/lens gain, $g$ & 3 & Camera-frame rate, $W_{\text {fps }}$ & 1000 fps \\
\hline Optical transmitting power, $P_{t}$ & $1.2 \mathrm{Watts}$ & Number of LEDs in the transmitter, $N_{\text {LEDs }}$ & $300(30 \times 10)$ \\
\hline Electron charge, $q$ & $1.6 \times 10^{-19} \mathrm{C}$ & Focal length of the camera lens, $f$ & $15 \mathrm{~mm}$ \\
\hline Equivalent noise/electronic bandwidth, $B$ & $2 \mathrm{MHz}$ & Image pixel size, $a$ & $7.5 \mu \mathrm{m}$ \\
\hline Distance between the left and right LED array, $D$ & $50 \mathrm{~cm}$ & Background current, $I_{b g}$ & $5100 \mu \mathrm{A}$ \\
\hline Noise bandwidth factor for a rectangular pulse, $I_{2}$ & 0.562 & Size of the LED, $L_{\text {size }}$ & $15.5 \times 5.5 \mathrm{~cm}$ \\
\hline Amplifier current, $I_{a m p} m$ & $5 \mathrm{pA}$ & Resolution of image, $w$ & $512 \times 512 \mathrm{pixels}$ \\
\hline Data rate of system, $R_{b}$ & $500 \mathrm{bps}$ & & \\
\hline
\end{tabular}

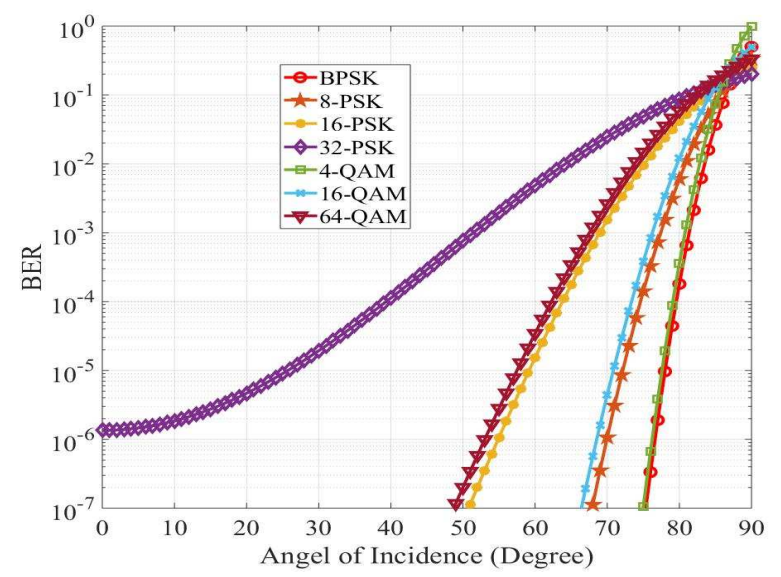

Fig. 4. BER vs AoI for different modulation schemes.

the interplay among the various parameters of our system. We consider adaptive modulation scheme with options BPSK, M-PSK, and M-QAM modulation with the constellation size, $M=\{4,8,16,32,64\}$, i.e., BPSK, 8-PSK, 16-PSK, 32PSK, 4-QAM, 16-QAM, 64-QAM. Target BER is set to $10^{-4}$ and $10^{-5}$ for performance comparison to be compliant with uRLLC requirements. All the results are generated considering the parameters in Table II.

We start by comparing the BER performance at different inter-vehicular distances and for different AoIs. The results are presented in Fig. 3 and Fig. 4, respectively, which show the achieved BER for the different modulation schemes. In this evaluation, we do not vary the distance and AoI at the same time. In Fig. 3, we change the distance from $0 \mathrm{~m}$ to $150 \mathrm{~m}$ by keeping the AoI at $60^{\circ}$, whereas in Fig. 4, we vary the AoI between $0^{\circ}$ to $90^{\circ}$ while keeping distance fixed at $50 \mathrm{~m}$. We note that the same BER can be achieved at different distances and AoIs by using different modulation schemes. Fig. 3 shows that 32-PSK satisfies target BER $\left(10^{-4}\right)$ up to $40 \mathrm{~m}$ and for 64-QAM, it is satisfied at $52 \mathrm{~m}$. At smaller distance, higher order modulation can be employed because of the higher SNR

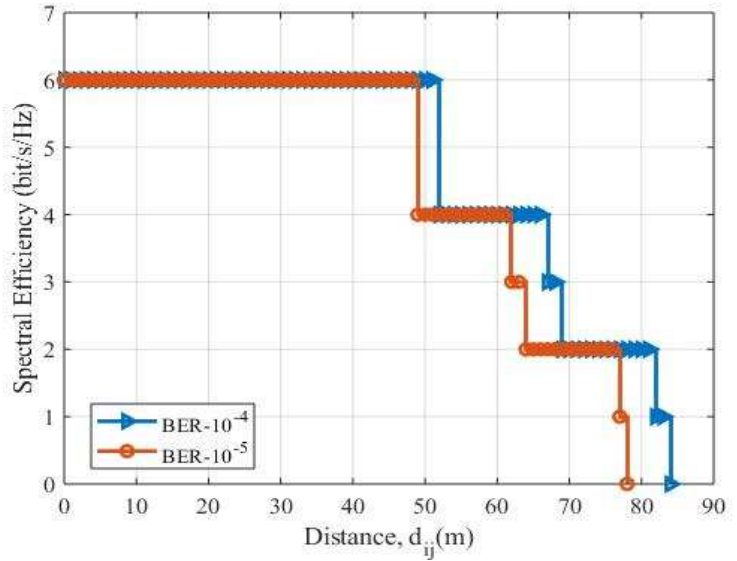

Fig. 5. Comparison of spectral efficiency vs distance at target BER of $10^{-4}$ and $10^{-5}$.

level. Similarly in Fig. 4, target BER $\left(10^{-4}\right)$ is satisfied at $38^{\circ}$ and $62^{\circ}$ for 32-PSK and 64-QAM. Because at the narrower AoI, the strength of light beam on the image sensor is strong which increases channel power gain. So, at shorter distance and narrower AoI, the modulation order will be higher, i.e., higher spectral efficiency, due to higher SNR at the receiver.

The spectral efficiency improvements offered by the proposed system are illustrated in Fig. 5 and Fig. 6 for two different target BERs $\left(10^{-4}\right.$ and $\left.10^{-5}\right)$. In this comparison, we explore the BER performance at different modulations by varying inter-vehicular distance (Fig. 5) and AoI (Fig. 6) and then choosing the highest spectral efficiency from all the available schemes that satisfy the target BER requirement. From Fig. 5, we see that we can support spectral efficiency of $6 \mathrm{bits} / \mathrm{s} / \mathrm{Hz}$ when the inter-vehicle distance is up to $48 \mathrm{~m}$ (for $\mathrm{BER}=10^{-5}$ ) and $52 \mathrm{~m}\left(\right.$ for BER $=10^{-4}$ ). From Fig. 6, we can notice that for 64 QAM, the spectral efficiency is $6 \mathrm{bits} / \mathrm{s} / \mathrm{Hz}$ when the AoI is $57^{\circ}$ (for BER $=10^{-5}$ ) and $62^{\circ}$ (for BER = $\left.10^{-4}\right)$. Similarly, our scheme achieves a spectral efficiency of $4 \mathrm{bits} / \mathrm{s} / \mathrm{Hz}$ from $48 \mathrm{~m}$ to $62 \mathrm{~m}$ and $57^{\circ}$ to $71^{\circ}, 52 \mathrm{~m}$ to 66 $\mathrm{m}$ and $62^{\circ}$ to $73^{\circ}$ for BER of $10^{-5}, 10^{-4}$, respectively. We 


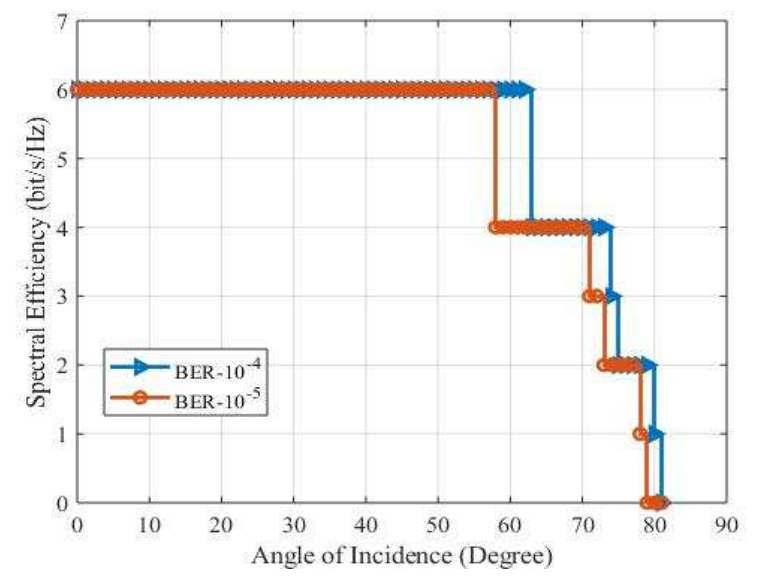

Fig. 6. Comparison of spectral efficiency vs AoI at target BER of $10^{-4}$ and $10^{-5}$.

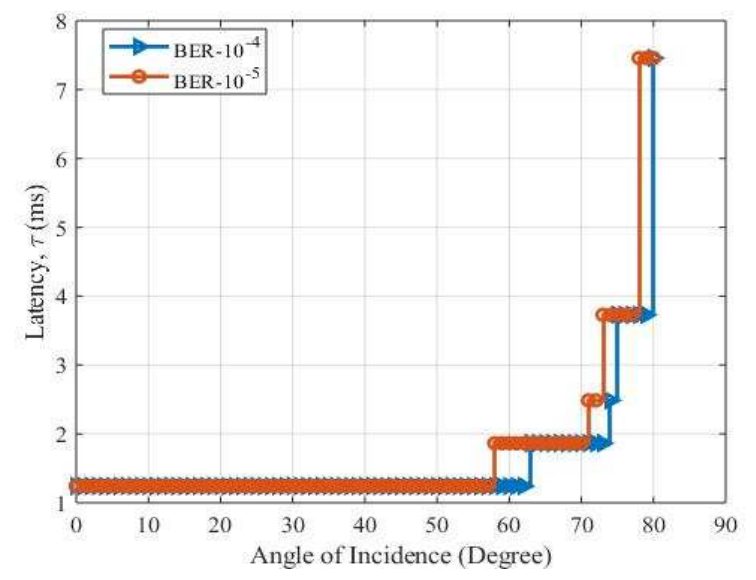

Fig. 7. Comparison of latency vs AoI at target BER of $10^{-4}$ and $10^{-5}$.

observe that lower distance and narrower AoI help achieving higher spectral efficiency. This is due to the higher gain at the receiver. We can conclude that the spectral efficiency of the adaptive system is up to six times than that of the system using BPSK. Please note that the above evaluation is ideal since it assumes that the modulation level is perfectly adapted and the probability of error is known beforehand and is accurate.

Finally, we estimate the capacity of our proposed system when the image sensor captures images with resolution of $512 \times 512$ pixels and the inter-vehicular distance is $50 \mathrm{~m}$. We evaluate the channel capacity using the spectral efficiency of Fig. 6. Then, we compute the transmission latency for the transmission of packets with size 5 kbits. The achieved latency by our adaptive system is shown for two different target BERs, i.e., $10^{-4}$ and $10^{-5}$, when AoI changes from $0^{\circ}$ to $90^{\circ}$ in Fig. 7. This evaluation shows that our system can achieve the latency of uRLLC, which is around $1 \mathrm{~ms}$ at $60^{\circ}$ of AoI. The latency increases for wider AoI as the light beam strength reduces with the increase of AoI of lights on the image sensor. Hence, the vehicular OCC model can ensure uRLLC by providing $1 \mathrm{~ms}$ latency and satisfying the reliability, i.e., $10^{-4}$ and $10^{-5}$ bit error probability, requirements.

\section{CONCLUSION}

In this paper, the performance of adaptive modulation has been analyzed for automotive vehicular uRLLC considering OCC. The latency is modelled based on the capacity of the vehicular OCC while considering the transmission latency only. Further, the BER performance is studied for various sets of the AoI and inter-vehicular distance. In our system, the spectral efficiency of vehicular OCC is adjusted adaptively using adaptive modulation which ensures reliability by maintaining the BER to a pre-determined target value. We carried our simulations to get an understanding of how to adjust the employed modulation scheme as well as AoIs so that it meets the BER requirements. Interestingly, the proposed model provides about $7 \mathrm{~ms}$ latency while satisfying the reliability requirement of $10^{-4}$ or $10^{-5}$ when the AoI is varied between $0^{\circ}$ to $90^{\circ}$.

\section{REFERENCES}

[1] A. E. Fernandez and M. Fallgren, "5GCAR scenarios, use cases, requirements and KPIs," Fifth Generation Communication Automotive Research and innovation, Tech. Rep. D2.1, Aug. 2017.

[2] S. Motoyama, T. Ohta, T. Watanabe, and Y. Ito, "Development of lane departure warning system," Proc. ITS World Congress, Turin, Italy, Nov. 2000

[3] J. Tjonnas and T. A. Johansen, "Stabilization of Automotive Vehicles Using Active Steering and Adaptive Brake Control Allocation," IEEE Trans. on Control Sys. Technol., vol. 18, no. 3, pp. 545-558, May 2010.

[4] S. Samarakoon, M. Bennis, W. Saad, and M. Debbah, "Distributed federated learning for ultra-reliable low-latency vehicular communications," [Online] Avaiable: https://arxiv.org/pdf/1807.08127.pdf

[5] M. Bennis, M. Debbah, and H. V. Poor, "Ultra-reliable and low-latency communication: Tail, Risk and Scale," Proc. the IEEE, vol. 106, no. 10, pp. 1834-1853, Oct. 2018.

[6] M. I. Ashraf, C.-F. Liu, M. Bennis, and W. Saad, "Towards low-latency and ultra-reliable vehicle-to-vehicle communication," Proc. European Conf. Net. and Commun., Oulu, Finland, Jun. 2017.

[7] W. Sun, E. G. Strom, F. Brannstrom, Y. Sui, and K. C. Sou, "D2Dbased V2V communications with latency and reliability constraints," Proc. IEEE GlobeCom Workshops, Austin, TX, Dec. 2014.

[8] M. M. K. Tareq, O. Semiari, M. A. Salehi, and W. Saad, "Ultra reliable, low latency vehicle-to-infrastructure wireless communications with edge computing," Proc. IEEE GlobeCom, Abu Dhabi, UAE, Dec. 2018.

[9] W. Shi and S. Dustdar, "The promise of edge computing," Computer, vol. 49, no. 5, pp. 78-81, May 2016.

[10] L. Zeng et al., "High data rate multiple input multiple output (MIMO) optical wireless communications using white LED lighting," IEEE J. Sel. Areas Commun., vol. 27, no. 9, pp. 1654-1662, Dec. 2009.

[11] R. Mesleh, H. Elgala, and T. D. C. Little, "A novel method to mitigate LED nonlinearity distortions in optical wireless OFDM systems," Proc. OFC/NFOEC, Anaheim, CA, Mar. 2013.

[12] A. Ashok, S. Jain, M. Gruteser, N. Mandayam, W. Yuan, and K. Dana, "Capacity of screen-camera communications under perspective distortions," Pervasive Mob. Comput., no. 16, pp. 239-250, Jan. 2015.

[13] T. Yamazato et al., "Image-sensor-based visible light communication for automotive applications," IEEE Commun. Mag., vol. 52, no. 7, pp. 88-97, Jul. 2014

[14] I. Takai, T. Harada, M. Andoh, K. Yasutomi, K. Kagawa, and S. Kawahito, "Optical vehicle-to-vehicle communication system using LED transmitter and camera receiver," IEEE Photon. J., vol. 6, no. 5, pp. 1-14, Oct. 2014.

[15] J. Kahn and J. Barry, "Wireless infrared communications," Proc. the IEEE, vol. 85, no. 2, pp. 265-298, Feb. 1997.

[16] T. Komine and M. Nakagawa, "Fundamental analysis for visible-light communication system using LED lights," IEEE Trans. on Consumer Electronics, vol. 50, no. 1, pp. 100-107, Feb. 2004.

[17] A. Goldsmith, "Wireless Communications," Cambridge University Press, 2005. 